\title{
On sediment transport under dam-break flow
}

\author{
By DAVID PRITCHARD AND ANDREW J. HOGG \\ Centre for Environmental and Geophysical Flows, School of Mathematics, University of Bristol, \\ University Walk, Bristol BS8 1TW, UK
}

(Received 5 August 2002 and in revised form 23 August 2002)

We present exact solutions for suspended sediment transport under one-dimensional dam-break flow, both over a dry bed and into a small depth of tail water. We explicitly calculate the suspended sediment concentration, including erosion and deposition, and investigate the effect of varying the erosional and depositional models employed. These solutions offer insight into sediment transport processes under floods or sheet flow events, and we also discuss their application as test-bed solutions for the validation of numerical models.

\section{Introduction}

Dam-break flows, which represent the sudden release of fluid from behind a vertical barrier, provide the simplest available model for a number of important phenomena, such as break-out floods, sheet flow events and the formative stages of lahars or debris flows. An idealized configuration for dam-break flow is shown in figure 1: the barrier at $\hat{x}=0$ divides fluids of different depths $\hat{h}_{0}$ and $\hat{h}_{2}$, until at time $\hat{t}=0$ the barrier is instantaneously removed, and fluid floods into the shallower region. The classical descriptions of these flows obtained by Ritter (1892) and by Stoker (1957) are among the simplest non-trivial solutions of the shallow-water equations, and may also be interpreted as a limiting form of constant-flux gravity current (Gratton \& Vigo 1994). They are not of merely mathematical interest, as they have been confirmed experimentally to provide a reasonably good description of the flow once transients associated with the initial release have died down (Stansby, Chegini \& Barnes 1998).

Recent studies by Capart \& Young (1998) and by Fraccarollo \& Capart (2002) have considered dam-break flows as agents of sediment transport. Both studies investigated flow over an erodible bed consisting of loose coarse sediment which could be mobilized en masse as bedload: they found that the flow forms an erosional bore, which advances digging a uniform scour pit beneath it and pushing the eroded sediment before it as a steep debris snout. The current paper may be regarded as complementary to these studies, and deals with the rather different sedimentary regime in which the bed consists of fine or cohesive material which, once a threshold shear stress is exceeded, is entrained into the water column and then transported in suspension. Our interest is in characterizing the quantity of sediment which such flows may mobilize and transport.

A secondary motivation for this work is computational. The shallow-water equations are among the principal tools employed in numerical studies of sediment transport processes and morphodynamics, and important morphodynamical flows frequently involve features such as internal bores and moving shoreline boundaries which present a significant challenge to the accuracy and stability of numerical schemes. A variety of analytical 'test-bed' solutions, of which dam-break flows are 


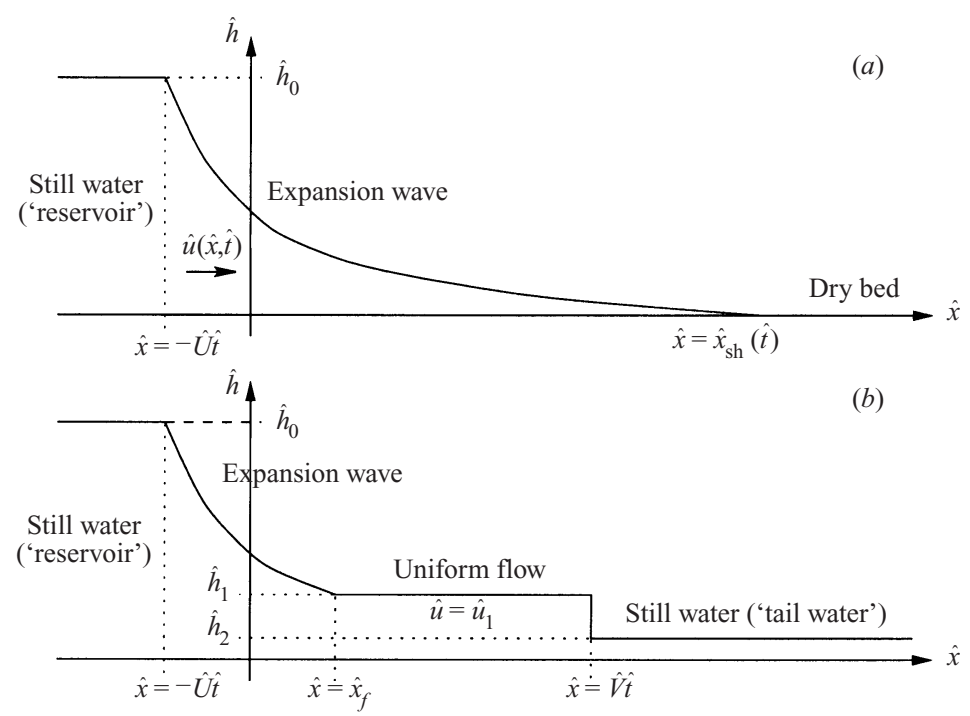

Figure 1. Schematics of the $(a)$ Ritter and $(b)$ Stoker solutions for dam-break flow on a horizontal bed.

perhaps the simplest and most popular, are used to validate the hydrodynamic components of such schemes. However, there is still a need for exact solutions which may be used to verify the accuracy of the corresponding predictions of sediment transport.

In $\S 2$, we describe the shallow-water model which we employ to describe sediment transport. In $\S 3$ we construct and discuss exact solutions, and in $\S 4$ we comment on the applications of our results.

\section{The shallow-water equations}

The one-dimensional shallow-water equations for flow over a horizontal bed may be derived from the Euler equations under the assumption that the characteristic horizontal length scale of the flow is much greater than the characteristic vertical length scale. In their non-dimensional form, the equations for the conservation of fluid mass and momentum have the form

$$
\frac{\partial h}{\partial t}+\frac{\partial}{\partial x}(u h)=0, \quad \frac{\partial u}{\partial t}+u \frac{\partial u}{\partial x}+\frac{\partial h}{\partial x}=0,
$$

where $h$ represents the depth of water and $u$ is a depth-averaged horizontal velocity. The variables here have been non-dimensionalized with respect to a length scale $\hat{h}_{0}$ corresponding to the initial height of the dam and a velocity scale $\hat{U}=\left(\hat{g} \hat{h}_{0}\right)^{1 / 2}$, where $\hat{g}$ is the acceleration due to gravity; they are given by

$$
h=\hat{h} / \hat{h}_{0}, \quad x=\hat{x} / \hat{h}_{0}, \quad u=\hat{u} / \hat{U}, \quad t=\hat{t} \hat{U} / \hat{h}_{0} .
$$

Throughout, carets denote dimensional quantities; dimensionless quantities are unadorned.

In dimensional form, the equation for the conservation of sediment transported as a dilute well-mixed suspension is given by

$$
\frac{\partial \hat{c}}{\partial \hat{t}}+\hat{u} \frac{\partial \hat{c}}{\partial \hat{x}}=\frac{1}{\hat{h}}\left(\hat{q}_{e}-\hat{q}_{d}\right),
$$


where $\hat{c}$ represents the mass concentration of suspended sediment and $\hat{q}_{e}$ and $\hat{q}_{d}$ are, respectively, the mass erosion and mass deposition fluxes which represent exchanges between the water column and the bed. In this formulation, we have assumed that the diffusive transport of particles in the horizontal direction is negligible compared to advective transport. We will also assume that the mass exchange fluxes may be expressed in terms of the vertically averaged quantity $\hat{c}$ rather than of the concentration of suspended sediment near the bed. This is formally valid when vertical mixing in the water column is sufficiently intense to maintain a vertically uniform concentration profile, in other words in the limit of infinite friction velocity. For finite friction velocities, the concentration profile is both vertically non-uniform and time-dependent, with a characteristic 'response time' which has been investigated for steady flows by Stansby \& Awang (1998). However, the approximation which we make here is well-established as the standard method by which empirical deposition formulae may be incorporated into a depth-averaged model: like the depth-averaged model itself, it provides an informative leading-order description of the system.

In general, the quantities $\hat{q}_{e}$ and $\hat{q}_{d}$ are functions of both the fluid velocity $\hat{u}$ and the suspended sediment concentration $\hat{c}$. In this paper, we consider the following form for the erosion rate $\hat{q}_{e}$ :

$$
\hat{q}_{e}(\hat{u})= \begin{cases}\hat{m}_{e}\left(\frac{\hat{u}^{2}}{\hat{u}_{e}^{2}}-1\right)^{n} & \text { for }|\hat{u}| \geqslant \hat{u}_{e} \\ 0 & \text { for }|\hat{u}|<\hat{u}_{e} .\end{cases}
$$

This model is commonly used to describe the erosion of sediment from a cohesive bed (Teisson et al. 1993) or from a bed of fine cohesionless material (Dyer \& Soulsby 1998), where some critical shear stress, corresponding to a velocity $\hat{u}_{e}$, must be exceeded in order to break up the bed and entrain particles. The quantity $\hat{m}_{e}$ is a dimensional mass erosion rate, while $n$ is a dimensionless exponent, typically in the range $n=1$ to $n=3.5$. We note that the approach described in this paper may readily be applied to other forms of the expression $\hat{q}_{e}(\hat{u})$.

A number of expressions have been proposed to describe the deposition of cohesive sediment at low flow rates (Teisson et al. 1993). However, as we will demonstrate, we need only consider deposition when $\hat{u}>\hat{u}_{e}$, and in this regime there are two possible models: either $\hat{q}_{d}=\hat{w}_{s} \hat{c}$, where $\hat{w}_{s}$ represents the settling velocity of the largest flocs which can survive the near-bed turbulence; or turbulence is too strong for flocculated matter to settle at all, and $\hat{q}_{d}=0$. It is often postulated that $\hat{q}_{d}=0$ when velocities are high enough to erode sediment, but Sanford \& Halka (1993) have demonstrated that this assumption may not be appropriate to describe sedimentary processes outside the laboratory. For this reason, and in order to make our results applicable to cohesionless particles with constant settling velocity $\hat{w}_{s}$, we will consider both forms of $\hat{q}_{d}$.

We can now define a reference concentration $\hat{C}=\hat{m}_{e} / \hat{w}_{s}$ (in the case $\hat{q}_{d}=0$, we may set $\hat{C}=\hat{m}_{e} / \hat{U}$ instead), and non-dimensionalize equation (2.3) by defining $c=\hat{c} / \hat{C}$, $q_{e}=\hat{q}_{e} / \hat{m}_{e}$ and $q_{d}=\hat{q}_{d} / \hat{m}_{e}$. It is also helpful to rewrite equation (2.3) in Lagrangian terms. We consider the concentration of sediment $c_{\mathrm{L}}(t)$ in a fluid 'parcel' with position $x_{\mathrm{L}}(t)$, depth $h_{\mathrm{L}}(t)$ and velocity $\mathrm{d} x_{\mathrm{L}} / \mathrm{d} t=u_{\mathrm{L}}(t)$. In this Lagrangian framework, equation (2.3) is then equivalent to

$$
\frac{\mathrm{d} c_{\mathrm{L}}}{\mathrm{d} t}=\frac{E}{h_{\mathrm{L}}}\left[q_{e}\left(u_{\mathrm{L}}\right)-q_{d}\left(c_{\mathrm{L}}\right)\right],
$$


where $E=\hat{w}_{s} / \hat{U}$ is a non-dimensional bed exchange rate representing the ratio of hydrodynamic to sedimentary time scales. The degenerate initial condition for dambreak flows allows us to eliminate $E$ from the problem by the further rescaling $x \mapsto E^{-1} x$ and $t \mapsto E^{-1} t$; in subsequent sections, this is presumed to have been done.

\section{Solutions for sediment transport}

Over a horizontal bed, the dam-break solutions have the form of simple waves (Stoker 1957). The most elementary case is Ritter's solution for flow over a dry horizontal bed, $h_{2}=0$. In this case (figure $1 a$ ), the flow field has the form of an expansion wave, analagous to a rarefaction wave in gas dynamics. When the region $x>0$ initially contains still fluid of depth $h_{2}>0$, the simple wave solution involves a rightwards-propagating bore as well as an expansion wave (see figure $1 b$ ).

\subsection{Ritter solution for flow over a dry bed}

\subsubsection{Hydrodynamic solution}

The expansion-wave solution due to Ritter (1892) has the form

$$
h(x, t)=\frac{1}{9}\left(2-\frac{x}{t}\right)^{2}, \quad u(x, t)=\frac{2}{3}\left(1+\frac{x}{t}\right)
$$

in the region $-1 \leqslant x / t \leqslant 2$.

We may readily describe this flow in Lagrangian terms. We consider a fluid parcel at $x=x_{\mathrm{L}}(t)$ : its velocity is given by $\mathrm{d} x_{\mathrm{L}} / \mathrm{d} t=\frac{2}{3}\left(1+x_{\mathrm{L}} / t\right)$, and thus $x_{\mathrm{L}}(t)=2 t+B t^{2 / 3}$ for some constant $B$ which labels the parcel. This leads immediately to

$$
u_{\mathrm{L}}(t)=\frac{\mathrm{d} x_{\mathrm{L}}}{\mathrm{d} t}=2+\frac{2}{3} B t^{-1 / 3}, \quad h_{\mathrm{L}}(t)=h\left(x_{\mathrm{L}}(t), t\right)=\frac{1}{9} B^{2} t^{-2 / 3} .
$$

When considering sediment transport, it is natural to relate the labelling quantity $B$ to $t_{e}$, the time at which the velocity of the fluid element attains the critical velocity for erosion. We find that $B\left(t_{e}\right)=\frac{3}{2}\left(u_{e}-2\right) t_{e}^{1 / 3}$, where $u_{e}=\hat{u}_{e} / \hat{U}$ is the dimensionless threshold velocity for erosion. We note that we require $u_{e}<2$ in order for erosion to occur, and thus that $B<0$ for all fluid parcels, so each parcel accelerates asymptotically towards a maximum velocity $u=2$. Hence with the initial condition $c=0$ everywhere, non-zero concentrations can only occur in regions where $u>u_{e}$ : this justifies our previous statement that we need only consider $q_{d}$ in this velocity range.

\subsubsection{Constructing solutions for the concentration field}

Under the flow defined by equations (3.2), equation (2.5) becomes

$$
\frac{\mathrm{d} c_{\mathrm{L}}}{\mathrm{d} t}=\frac{9 t^{2 / 3}}{B^{2}}\left[q_{e}\left(u_{\mathrm{L}}(t)\right)-\epsilon c_{\mathrm{L}}(t)\right] \quad \text { for } t>t_{e},
$$

where $\epsilon=0$ for $q_{d}=0$ and $\epsilon=1$ for $q_{d}=c$. The initial condition is given by $c_{\mathrm{L}}\left(t_{e}\right)=0$.

This equation may be integrated to give

$$
c_{\mathrm{L}}(t)=A(t) \int_{t_{e}}^{t} \frac{9 z^{2 / 3}}{A(z)} \frac{q_{e}\left(u_{\mathrm{L}}(z)\right)}{B^{2}} \mathrm{~d} z, \quad \text { where } \quad A(t)=\exp \left[-\frac{27 \epsilon}{5 B^{2}} t^{5 / 3}\right] .
$$

The integral cannot in general be expressed in terms of elementary functions, but is easily evaluated using standard packages such as MAPLE. 

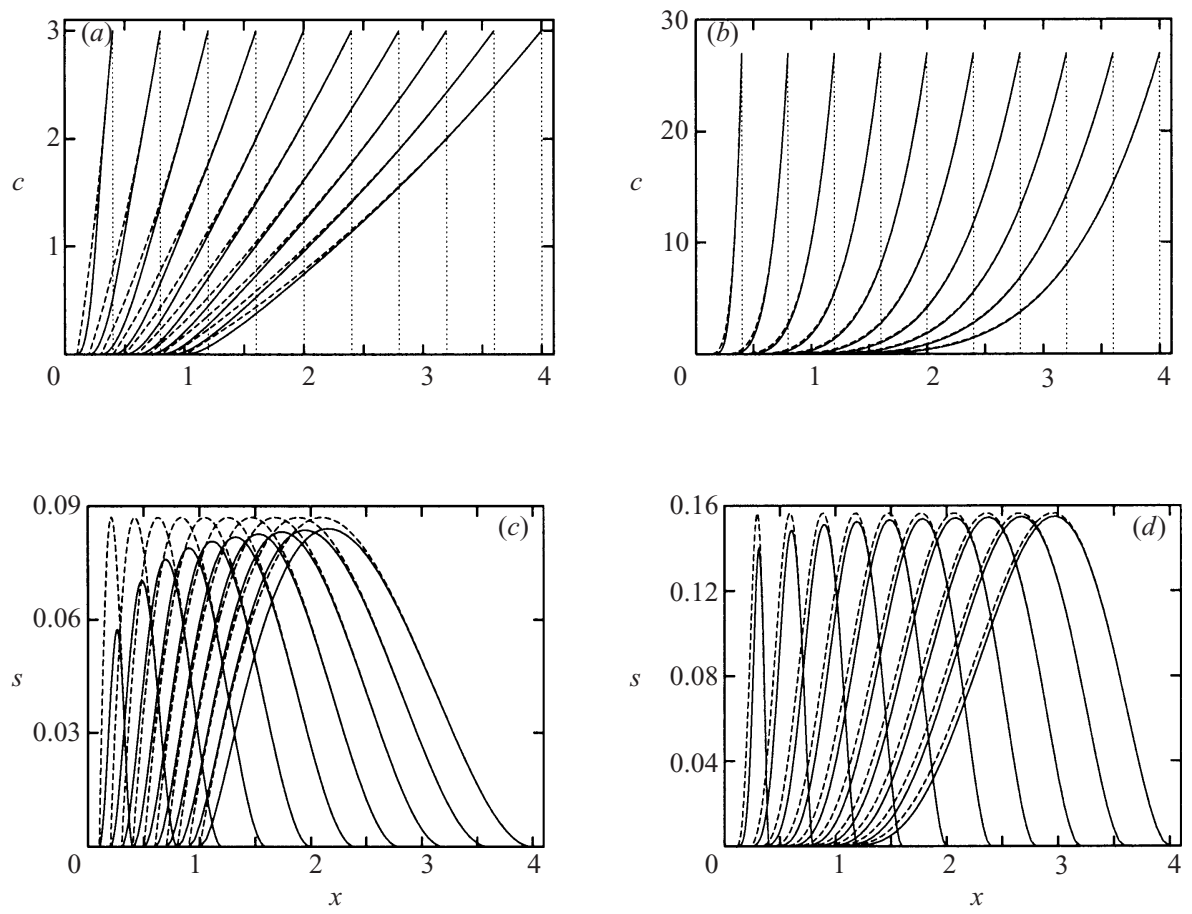

FigURE 2. Solutions for sediment transport with $q_{d}=c$ and $u_{e}=1$. Successive plots at $t=0.2$ to 2.0. Concentration field $c$ for $(a) n=1$ and $(b) n=3$; sediment load $s=c h$ for $(c) n=1$ and (d) $n=3$. Solid lines show exact solutions, dashed lines show solutions with $c=c_{\text {eq }}$.

Finally, by substituting for $B$ in terms of $t_{e}$, and then for $t_{e}(x, t)$, given by

$$
t_{e}(x, t)=\frac{1}{t^{2}}\left[\frac{2}{3}\left(\frac{2 t-x}{2-u_{e}}\right)\right]^{3}
$$

we obtain the Eulerian concentration field $c(x, t)$.

Before discussing the solutions, it is helpful to define the quantity $c_{\mathrm{eq}}=q_{e}(u(x, t))$. In the case $q_{d}=c$, this quantity represents an instantaneous equilibrium between erosion and deposition in a fluid parcel, and it is straightforward to show that in any given parcel $c_{\mathrm{L}}(t) \rightarrow c_{\mathrm{eq}}\left(u_{\mathrm{L}}(t)\right)$ as $t \rightarrow \infty$. (In fact, the convergence is rather rapid, especially in the shallower parts of the flow, and this provides a useful means of evaluating $c(x, t)$ close to the front of the flow $x=x_{\mathrm{sh}}(t)$, where plotting programs may have difficulty handling the exponentials in equation (3.4).) Since $c_{\mathrm{eq}}(t)$ is clearly bounded above by the shoreline concentration $c_{\mathrm{sh}}=q_{e}(2)$, we obtain the result that when deposition occurs, concentrations must be bounded by $c=c_{\mathrm{sh}}$, even in very shallow water close to the front. Figure 2 illustrates this point.

\subsubsection{Examples of the solutions}

Figure 2 illustrates the solutions with continuous deposition for two values of $n$. The principal feature of the plot of concentration $c$ is the maximum at the front, where flow is fastest and has been erosional for longest. However, the sediment load $s=c h$ is highest in somewhat deeper water, and in fact tends strongly to zero near the front. As $n$ is increased, the shoreline concentration increases dramatically and the plots of $c$ become more concave, because of the enhanced erosion at higher 

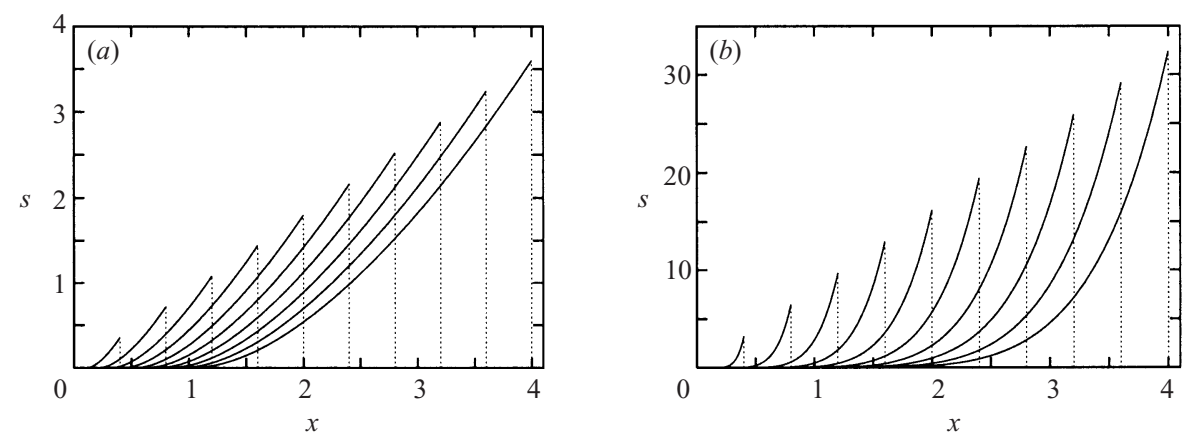

FIGURE 3. Solutions for sediment transport with $q_{d}=0$ and $u_{e}=1$. Successive plots at $t=0.2$ to 2.0 , showing sediment load $s=\operatorname{ch}$ for $(a) n=1$ and $(b) n=3$.

velocities. However, the overall sediment load increases much more slowly, because of the reduced erosion at low velocities. Because of the concavity of $c(x, t)$, the maximum of $s$ occurs rather closer to the front for higher $n$, and since velocities are higher there, the maximum sediment flux $q=c u h$, which describes the transport potential of the flow, is further enhanced for higher $n$.

Figure 3 illustrates the solutions when deposition is neglected entirely. In this case, the plots of $c$ are dominated by the unbounded maximum which develops at the front, and so we show only the plots of total sediment load $s=c h$. This quantity increases monotonically towards its value at the front $s=s_{\mathrm{sh}}$. By considering the sediment transport equation evaluated at $x=x_{\mathrm{sh}}$, we obtain the result

$$
\left.\left[\frac{\partial(\text { ch })}{\partial t}+\frac{\partial(\text { chu })}{\partial x}\right]\right|_{x_{\mathrm{sh}}}=\frac{\mathrm{d} s_{\mathrm{sh}}}{\mathrm{d} t}+\left.s_{\mathrm{sh}} \frac{\partial u}{\partial x}\right|_{x_{\mathrm{sh}}}=\frac{\mathrm{d} s_{\mathrm{sh}}}{\mathrm{d} t}+\frac{2}{3 t} s_{\mathrm{sh}}=q_{e}(2)
$$

and since $q_{e}(2)$ is a constant, and there is initially no sediment in suspension, we obtain

$$
s_{\mathrm{sh}}=\frac{3}{5} \operatorname{tq}(2) .
$$

The unboundedness of $c$ at the front is unphysical, since when concentrations become sufficiently high, the presence of suspended matter significantly affects the rheology of the flow, and the hydrodynamics must be modified accordingly (Iverson 1997). This may, of course, occur also for the solutions in which deposition is included, in which case it will depend on the value of $\hat{C}$ as well as on the criterion for the suspension to be regarded as dilute. However, a comparison between figure 3 and figure 2 suggests that including deposition must significantly alter any estimate which we are able to make of the time taken for such a flow to 'bulk up' in this manner.

\subsubsection{Net scour}

A final point which these solutions illustrate is the importance of deposition in controlling the net scour beneath the flow. We have carried out these calculations assuming that the total quantity of sediment eroded is sufficiently small that the bed geometry is not significantly altered. If the depth of scour, which is proportional to the amount of material eroded per unit length, can increase unboundedly, then this assumption will become invalid after sufficient time has passed.

We can quite readily estimate the total amount of sediment eroded. When deposition occurs, the concentration field is bounded above by $c_{\text {eq }}(u(x, t))$, and so the total 
quantity of sediment in suspension, $S(t)$, is bounded by

$$
S(t) \leqslant \int_{0}^{2 t} c_{\mathrm{eq}}(u(x, t)) h(x, t) \mathrm{d} x=t \int_{0}^{2} c_{\mathrm{eq}}(u(\xi)) h(\xi) \mathrm{d} \xi, \quad \text { where } \quad \xi=\frac{x}{t} .
$$

Since the length of the region from which material has been eroded also increases linearly in time, we may conclude that when deposition is allowed for, the depth of scour beneath the flow may not increase unboundedly, and so the solutions obtained here may remain formally consistent.

When deposition is neglected, we have no upper bound for the concentration field. However, we may write

$$
\frac{\mathrm{d} S}{\mathrm{~d} t}=\int_{0}^{2 t} q_{e}(u(x, t)) \mathrm{d} t=t \int_{0}^{2} q_{e}(u(\xi)) \mathrm{d} \xi,
$$

so $S(t) \propto t^{2}$, for any form of $q_{e}$. Hence the maximum depth of scour must increase at least linearly with time, and so when deposition is neglected, solutions for erosive dam-break flows which neglect the effect of scour on the bed must become inconsistent at large times.

\subsection{Stoker's solution and the effect of tail water}

Stoker's (1957) extension to Ritter's dam-break solution describes the propagation of a dam-break wave into a region of still 'tail water' of scaled depth $h_{2}<1$. The solution is illustrated in figure $1(b)$ : it consists of a bore propagating into the tail water with velocity $V$, behind which is a region of uniform flow with $u=u_{1}$ and $h=h_{1}$, which is itself linked by Ritter's expansion wave solution to the quiescent reservoir behind.

Given the downstream depth $h_{2}=\hat{h}_{2} / \hat{h}_{0}$, the quantities $V, h_{1}$ and $u_{1}$ may easily be deduced from the conservation of mass and momentum across the bore, and from the condition that velocity and surface elevation are continuous at $x=x_{f}$, where the expansion wave meets the region of uniform flow. We may express $V, h_{1}$ and $h_{2}$ as functions of $u_{1}$, and then invert the solutions numerically to obtain $u_{1}$, and hence $V$ and $h_{1}$, as functions of $h_{2}$. (For further details of the hydrodynamic solution, see Stoker 1957.) A feature of the solution is that it varies strongly at $h_{2}=0$, with

$$
u_{1} \sim 2-2^{7 / 4} h_{2}^{1 / 4}, \quad V \sim 2-2^{7 / 4} h_{2}^{1 / 4}, \quad h_{1} \sim 2^{3 / 2} h_{2}^{1 / 2} \quad \text { for } \quad h_{2} \ll 1,
$$

so even a small tail water depth has a dramatic effect on $u_{1}$ (see figure $5 a$ below).

This is of interest because of the possible presence of tail water in physical contexts such as a breakout flood entering a river valley, and also because a popular means of handling the shoreline condition for beach and dam-break problems is to impose a small notional water depth everywhere in the numerical domain, so that the shoreline becomes effectively a bore. It is therefore important to investigate the effects this may have on sediment transport.

We may express the flow field in Lagrangian form as we did for the Ritter solution, and construct solutions for sediment transport in exactly the same manner as before (see equations (3.3) and (3.4)). The algebraic details of this construction are rather lengthy and are not informative, and we therefore omit them here. However, certain features of the resulting sediment transport are worth calling attention to.

Figure 4 illustrates a typical solution, with the rather small tail water depth $h_{2}=0.01$. The main feature of the plots is the region of near-constant sediment concentration which coincides with the region of uniform flow. A fluid parcel may 

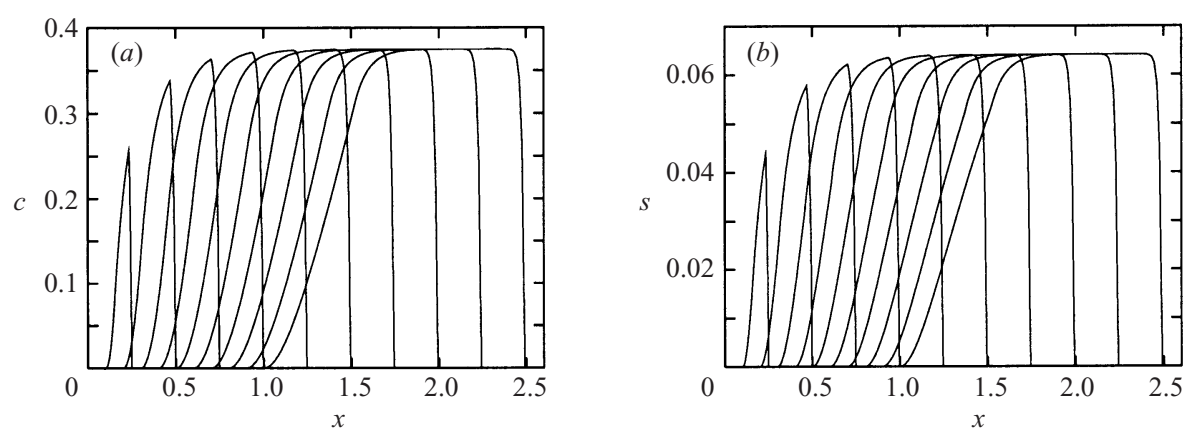

FIGURE 4. Solutions for sediment transport under Stoker's dam-break solution with $q_{d}=c, h_{2}=0.01$, $u_{e}=1$ and $n=1$. Successive plots at $t=0.2$ to 2.0: $(a)$ concentration field $c ;(b)$ sediment load $s=c h$ (compare with the Ritter solution, figures $2 a$ and $2 c$ ).

enter this region either from the expansion wave behind or through the bore from the tail water ahead; having entered, it remains in the region of uniform flow, and the concentration adjusts asymptotically to its equilibrium value in this region,

$$
c_{\mathrm{L}}(t)=c_{\mathrm{eq}}\left(u_{1}\right)-K \mathrm{e}^{-t / h_{1}},
$$

where $K$ is a constant of integration which depends on the initial position of the fluid parcel.

Figure 4(b) illustrates the effect the presence of tail water has on the total sediment load, and thus on the capacity of the flow to erode and transport sediment. Comparing figures $2(c)$ and $4(b)$, we observe that the difference between the sediment load for $h_{2}=0$ and $h_{2}=0.01$ is quite substantial: the maximum sediment load is reduced from $s=0.087$ to $s=0.064$, and the sediment-laden region extends only to $x=V t$ rather than to $x=2 t$. Figure 5 provides the explanation for this: it illustrates how the very rapid decrease in $u_{1}$ as $h_{2}$ increases from zero drives a strong decrease in $c_{\text {eq }}\left(u_{1}\right)$, and hence in both the sediment load and the sediment flux in the uniform region. The effect is amplified for higher $n$ by the stronger dependence of $c_{\text {eq }}$ on $u$.

These results indicate that considerable caution is required when numerically modelling sediment transport under flows with a moving shoreline, since if even a small bore develops it can lead to unexpectedly large changes in the sediment transport rate. As well as indicating the possibility of such errors, the solutions presented here offer a means to identify them by more thoroughly testing numerical schemes.

\section{Conclusions}

We have presented exact solutions which describe suspended sediment transport under dam-break flow. For dam-break flow over a dry bed, the concentration field increases monotonically towards the front, while the total sediment load has a maximum somewhat behind it. Conversely, for flow into quiescent tail water, a region of near-uniform sediment concentration rapidly develops behind the bore, corresponding to a local balance between the erosion and deposition of sediment. Furthermore, the maximum concentrations and maximum sediment load in the flow are both substantially reduced compared to the dry bed solution, for even a relatively small tail water depth. This suggests that, while the dry bed solution is the simplest prototype for many physically relevant flows, the insight provided by the Stoker solution is important in interpreting the dry bed results. 

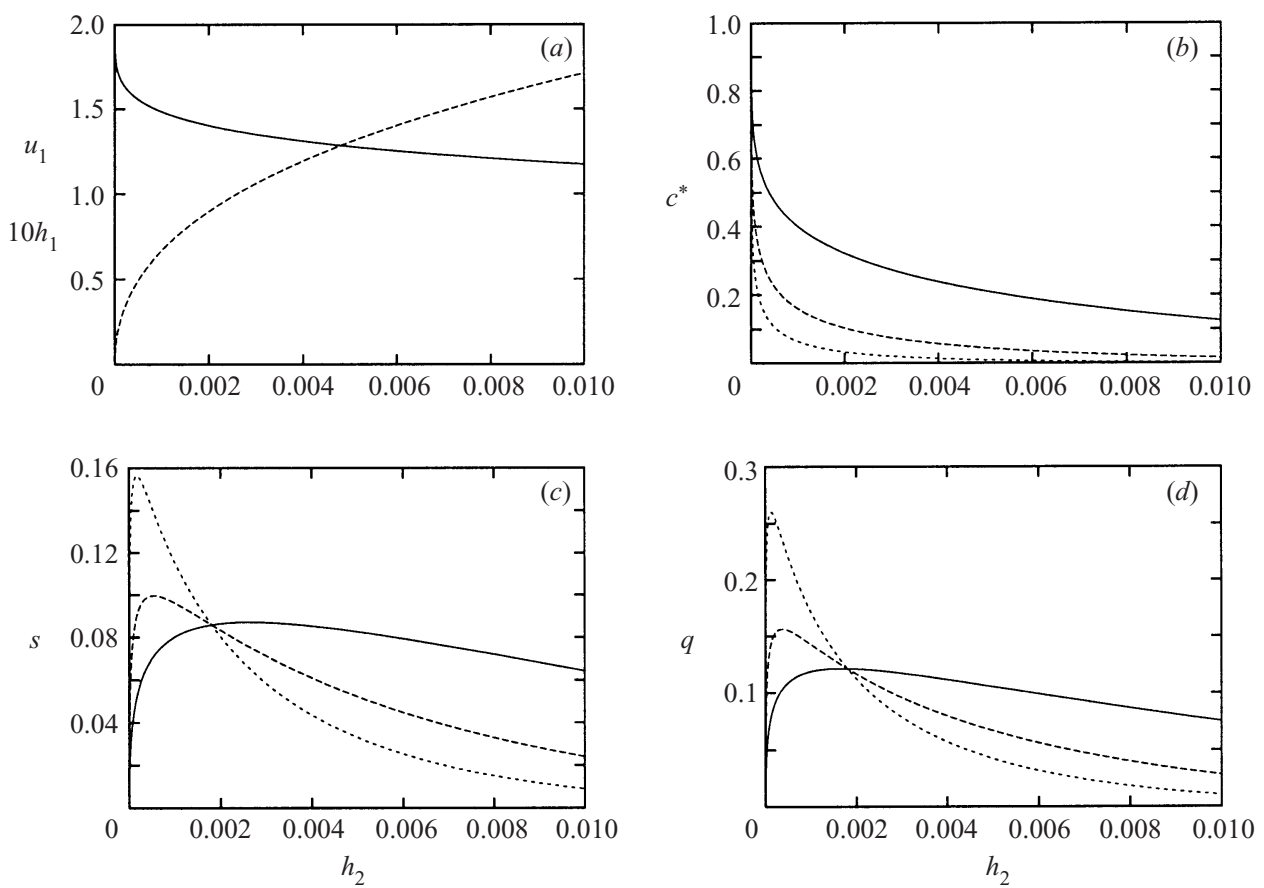

FIGURE 5. The effect of a small tail water depth $h_{2}$ on hydrodynamics and suspended sediment behind the bore. (a) Maximum velocity $u_{1}$ (solid line) and depth $h_{1}$ (dashed line; scaled by a factor of 10 for clarity) in the uniform region. $(b),(c)$ and $(d)$ Scaled equilibrium concentrations $c^{*}=c_{\mathrm{eq}}\left(u_{1}\right) / c_{\mathrm{eq}}(2)$; equilibrium sediment loads $s=h_{1} c_{\mathrm{eq}}\left(u_{1}\right)$; and equilibrium sediment fluxes $q=h_{1} u_{1} c_{\mathrm{eq}}\left(u_{1}\right)$, for $n=1$ (solid), $n=2$ (dashed), $n=3$ (dotted).

As well as indicating the sensitivity of sediment transport to the presence of tail water, our solutions illustrate how the quantity of sediment mobilized depends on the models employed for the erosion and deposition of suspended sediment, and the implications of this for the bulking up of the suspension into a debris flow. A secondary role for these solutions is as test-bed cases, which are easily reproduced and may be used to validate numerical schemes for predicting sediment transport within shallow-water models.

Throughout this study, we have employed the simplest available descriptions of sediment distribution in the water column. One extension which might yield interesting results is to consider in more detail the vertical distribution of suspended sediment (Stansby \& Awang 1998); another is to investigate how dam-break flows might fluidize a cohesive bed and transport material as a fluid mud layer, analagous to the non-cohesive bedload considered by Capart \& Young (1998) and by Fraccarollo \& Capart (2002). Both extensions lie beyond the scope of this paper. However, they are natural developments of the existing work in this area, and could provide further insight into problems such as the early stages of lahar formation.

The method used to construct the exact solutions presented here is easily applied to other problems in which the flow field may be expressed in Lagrangian terms. An immediate extension of the solutions described here is to the dam-break flow on a slope which has been used as a model for the swash that results from a breaking wave on a plane beach (Shen \& Meyer 1963), and work on this problem is currently underway. A similar approach has recently been applied to describe sediment transport under tidal flows on mudflats (Pritchard 2001). 
D. P. and A. J.H. acknowledge the financial assistance of EPSRC and HR Wallingford Ltd, and would like to thank two anonymous referees for their helpful suggestions.

\section{REFERENCES}

CAPART, H. \& Young, D. L. 1998 Formation of a jump by the dam-break wave over a granular bed. J. Fluid Mech. 372, 165-187.

Dyer, K. R. \& Soulsby, R. L. 1988 Sand transport on the continental shelf. Annu. Rev. Fluid Mech. 20, 295-324.

Fraccarollo, L. \& Capart, H. 2002 Riemann wave description of erosional dam-break flows. J. Fluid Mech. 461, 183-228.

Gratton, J. \& Vigo, C. 1994 Self-similar gravity currents with variable inflow revisited: plane currents. J. Fluid Mech. 258, 77-104.

Iverson, R. M. 1997 The physics of debris flows. Rev. Geophys. 35, 245-296.

Pritchard, D. 2001 Some problems in two-phase flow. PhD thesis, University of Bristol.

Ritter, A. 1892 Die Fortpflanzung der Wasserwellen. Z. Verein. Deutsch. Ing. 36, 947-954.

SANFord, L. P. \& HALKA, J. P. 1993 Assessing the paradigm of mutually exclusive erosion and deposition of mud, with examples from upper Chesapeake Bay. Mar. Geol. 114, 37-57.

Shen, M. C. \& Meyer, R. E. 1963 Climb of a bore on a beach. Part 3. Run-up. J. Fluid Mech. 16, $113-125$.

Stansby, P. K. \& Awang, M. A. O. 1998 Response time analysis for suspended sediment transport. J. Hydr. Res. 36, 327-338.

Stansby, P. K., Chegini, A. \& Barnes, T. C. D. 1998 The initial stages of dam-break flow. J. Fluid Mech. 374, 407-424.

Stoker, J. J. 1957 Water Waves: The Mathematical Theory with Applications. Interscience.

Teisson, C., Ockendon, M., Le Hir, P., Kranenburg, C. \& Hamm, L. 1993 Cohesive sediment transport processes. Coastal Engng 21, 129-162. 